\title{
Streamwise counter-rotating vortices generated by triangular leading edge pattern in flat plate boundary layer
}

\author{
S. M. Hasheminejad ${ }^{1, *}$, H. Mitsudharmadi ${ }^{2}$, S. H. Winoto ${ }^{3}$, K. B. Lua ${ }^{1}$, H. T. Low ${ }^{1}$ \\ ${ }^{1}$ Department of Mechanical Engineering, National University of Singapore, Kent Ridge, Singapore, Singapore \\ ${ }^{2}$ Reactive Flow Modeling Laboratory Group in Clean Combustion Research Center, King Abdullah University of \\ Science and Technology, Thuwal, Kingdom of Saudi Arabia \\ ${ }^{3}$ Faculty of Engineering, Tembalang Campus, Universitas Diponegoro, Semarang, Indonesia \\ *Corresponding author: E-mail: M.Hasheminejad@u.nus.edu \\ Tel.:+65-93733162
}

\begin{abstract}
A series of flow visualizations were conducted to qualitatively study the development of streamwise counter-rotating vortices over a flat plate induced by triangular patterns at the leading edge of a flat plate. The experiments were carried out for a Reynolds number based on the pattern wavelength $(\lambda)$ of 3080 . The results depict the onset, development and breakdown of the vortical structures within the flat plate boundary layer. Moreover, the effect of one spanwise array of holes with diameter of $0.2 \lambda(=3 \mathrm{~mm})$ was examined. This investigation was done on two different flat plates with holes placed at the location $x / \lambda=2$ downstream of the troughs and peaks. The presence of holes after troughs does not show any significant effect on the vortical structures. However, the plate with holes after peaks noticeably delays the vortex breakdown. In this case, the "mushroom-like" vortices move away from the wall and propagate downstream with stable vortical structures. The vortex growth is halted further downstream but start to tilt aside.
\end{abstract}

Keywords: Leading edge pattern, Streamwise counter-rotating vortices, Smoke-wire flow visualization, Flat plate boundary layer.

\section{Introduction}

Flow control is required in a lot of fluid engineering applications to improve the flow condition and thermal system efficiency. There are a wide variety of approaches for flow control. Some of them do not need any additional energy to control the flow and are known as passive methods. One of the most effective and feasible passive methods is to generate streamwise counter-rotating vortices within a boundary layer flow which promotes the exchange of momentum between the free stream and the flow near wall, resulting in the energizing of the boundary layer flow. This may delay the boundary layer separation, which is favorable in aerodynamics. Additionally, the heat transfer rate may also increase due to the mixing enhanced by the streamwise counter-rotating vortices particularly near the wall zone(Webb and Kim 2005). Vortex generators (VGs) are frequently utilized to generate streamwise vortices by making discontinuity and shear forces in the flow (Lin et al. 1991; Godard and Stanislas 2006). Many studies have examined and optimized the VGs in term of shape, size, orientation and configuration. In all studies, VGs may have shown increased aerodynamic lift but at the expense of increased drag, which is unfavorable. Another method used to produce streamwise vortices in the boundary layer is by attaching a serrated strips to the airfoil lower surface and near the leading edge (Soderman 1972). The results revealed that the leading edge serrations delay separated flow and increase maximum lift, if they are placed at a proper position to the leading edge. Moreover, the smaller serrations as well as the great enough serrations improved the performance. However, the leading edge serrations manifest drag penalty like for the case of vortex generators. Therefore, finding a passive flow control method utilizing streamwise counter-rotating vortices without any drag penalty is desirable for many industrial applications. Utilizing leading edge pattern is such a technique to generate streamwise counter-rotating vortices.

The idea of using leading edge pattern is actually inspired by nature. The combination of large sinusoidal tubercles and the high aspect ratio of flippers on humpback whales leads to an increase of the lift to drag ratio and enables it to do sharp banking turns, and to be more maneuverable compared to other species in spite of its massive body (Fish and Battle 1995; Fish et al. 2011). Wind tunnel experiments conducted by Miklosovich et al (2004) showed that the tubercles cause the flipper to have a greater stall angle and maximum lift coefficient. The result also shows that the lift coefficient gradually drops during the stall, in contrast to the lift coefficient which sharply drops in the absence of the tubercles. However, tubercles may adversely affect two-dimensional airfoils. Significant lift loss and drag rise may result from applying tubercles on two-dimensional airfoil (Stein and Murray 2005; Van Nierop et al. 2008). This contradiction is likely due to the absence of the tip effect in two-dimensional models. Johari et al. (2007) studied the role of the periodic sinusoidal protuberances on the leading edge of a two-dimensional airfoil. They showed that the flow separation occurs downstream of the troughs earlier than downstream of the protuberances. The results indicated that the protuberance amplitude was more influential than its wavelength on the airfoil performance. However, this 
conclusion is not in agreement with that of Hansen et al. (2011) who found that the wavelength was as effective as the amplitude. They stated that there is an optimal wavelength for each amplitude at which the airfoil performance reaches its maximum possible. Moreover, they introduced the locus of the maximum thickness on the airfoil profile as a parameter which should be taken into account. In fact, the appearance of the tubercles is more effective as the locus of the maximum thickness is placed further downstream. Further studies on modified two-dimensional airfoils at low Reynolds number show that, similar to three-dimensional ones, the lift gradually drops at stall angles while it still stays greater than that of the unmodified models at the post-stall angle. On the other hand, the pre-stall performance suffers from the appearance of the leading edge tubercles (Stein and Murray, 2005; Johari et al., 2007; Hansen et al., 2011; Miklosovic et al., 2007). Cranston et al. (2012) fabricated flat plates with saw-tooth serrations on the leading edge to examine the effect of the leading edge variation on the aerodynamic performance in absence of the curvature and thickness change effects. The experiments were performed in a range of low Reynolds numbers at angles of attack ranging from $0^{\circ}$ to $25^{\circ}$. It was shown that the serration size is more effective as the Reynolds number increases such that the larger serrations led to lift reduction at all angles of attack. All the above studies involved the generation of streamwise vortices within the boundary layer via leading edge variations which are functionally comparable to conventional vortex generators and delta-wings due to similar manner in vortex formation and characteristics (Hansen et al. 2011; Wei et al. 2015). To have a better picture of how the leading edge variations generate the streamwise vortices, a set of flow visualizations were carried out by Hasheminejad et al. (2014). Different geometrical patterns at the leading edge of a flat plate were examined at zero-pressure gradient condition. It was reported that immediately downstream of each trough, an upwash region including a pair of counter-rotating vortices is longitudinally developed. The vortices become diffused further downstream as a consequence of the disturbance amplification and eventually broken down into turbulence. The observations showed that the vortices shorten the laminar-turbulent region. This conclusion confirms the earlier observations in which the separation is delayed downstream of the trough of an airfoil fitted with protuberances (Johari et al. 2007; Hansen et al. 2011). In fact, the delay in separation is a consequence of transferring the momentum from the free stream into the boundary layer by means of the streamwise counter-rotating vortices.

Most of the aforementioned studies were focused on the role of the leading edge protrusions on the aerodynamic characteristics, while the mechanism of the appearance and development of this type of streamwise vortices was not as thoroughly considered. It is also interesting that there are similarities between this type of streamwise vortices and those produced by some other passive devices like vortex generators (Velte et al. 2007; Aider et al. 2008; Nadesan et al. 2014) or by centrifugal forces like Görtler vortices (Winoto and Low 1991; Winoto et al. 2005; Tandiono et al. 2009; Budiman et al. 2014). Therefore, the purpose of this work is to study, by means of flow visualization, the development of streamwise counter-rotating vortices induced by triangular pattern on the leading edge of a flat plate, as shown in Fig 1, under zero-pressure gradient condition. The development of the vortices is controlled by a simple technique which uses small holes at specific locations downstream of the pattern troughs and peaks, each in separate plates.

\section{Description of experiment}

Three plexiglass plates of $158 \mathrm{~mm}$ (width in spanwise direction) x $660 \mathrm{~mm}$ (length in streamwise direction) x $3 \mathrm{~mm}$ (thickness) were used in this work. A triangular pattern which consisted of six side-by-side triangles with no free space between was fabricated on the leading edge of the plates. Each triangle had a $15 \mathrm{~mm}$ base (or wavelength $(\lambda)$ ) and a height (or amplitude A) of $7.5 \mathrm{~mm}(=\lambda / 2)$ as shown in Fig 1. The leading edge of the plates was made tapered by machining with acute angle of $20^{\circ}$ to avoid the flow separation that could form the separation bubble at the leading edge of the flat plate model.. The test plates were similar to those used in (Hasheminejad et al. 2014); however, one spanwise array of $0.2 \lambda(=3 \mathrm{~mm})$ diameter holes were drilled on two out of three plates at location of $\mathrm{x} / \lambda=2$ downstream of the leading edge. For convenience, the plate with holes at downstream of troughs is called "plate A" while that plate with holes at downstream of the peaks is called "plate B" (Fig 1). In addition the third plate with no hole will be specified as "reference plate". To have a smooth surface and to diminish the reflection for the flow visualization, all plates were covered by a black matt film.

The experiments were carried out for zero plate angle of incidence in a small open-circuit low-speed wind tunnel with a maximum flow speed of $35 \mathrm{~m} / \mathrm{s}$. The free-stream turbulence intensity of the wind tunnel is less than $0.25 \%$ for a velocity range of $1-4 \mathrm{~m} / \mathrm{s}$. The wind tunnel has a contraction ratio of $9.8: 1$ followed by a test section $160 \mathrm{~mm}$ by 160 $\mathrm{mm}$. Since the plate span is as wide as the test section span, the model can be considered as two-dimensional model.

To visualize the vortices over the plates, a smoke-wire visualization aided by a green light laser source were used. The smoke-wire apparatus consisted of a Nickel-Chromium wire diameter of $0.193 \mathrm{~mm}$, a pressurized container holding paraffin oil mounted on the top of the test section and a manual traversing mechanism as described by Nadesan et al. (2014) and Budiman et al. (2014). 


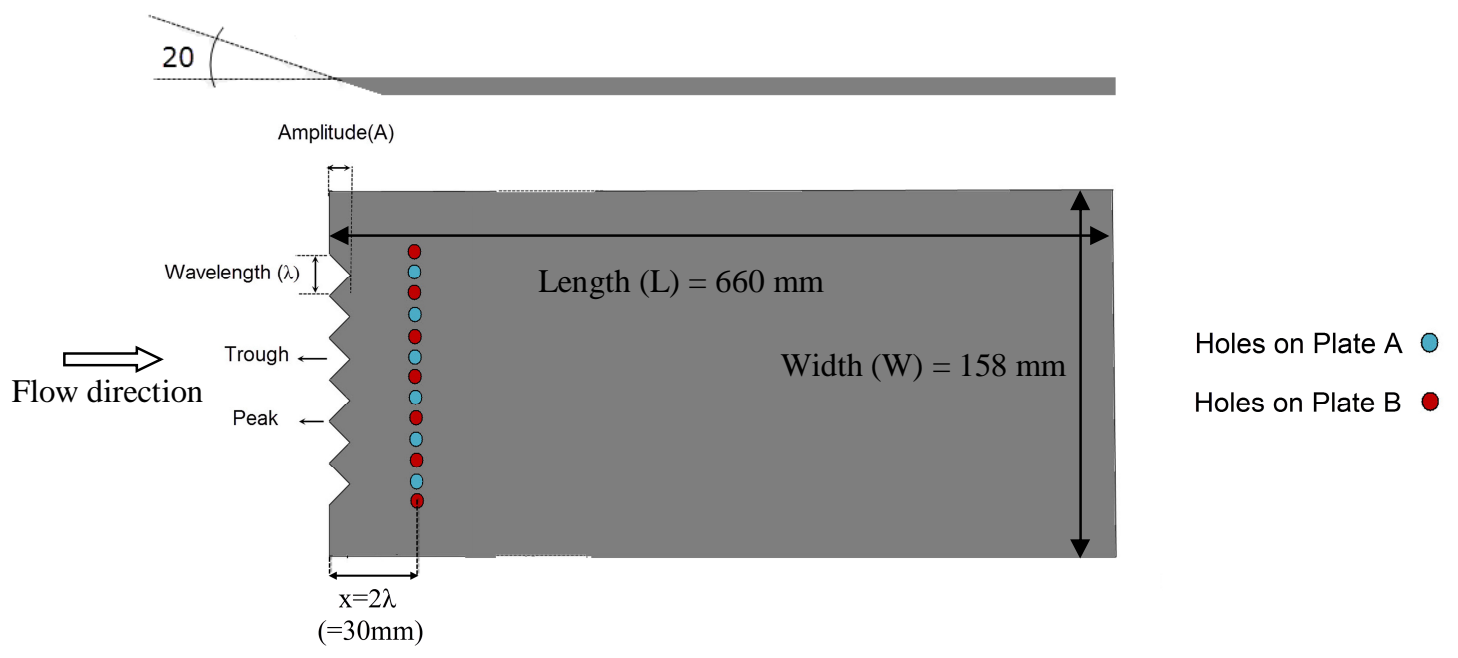

Fig. 1 Schematic of flat plate with leading edge pattern (a) side view and (b) Top view of positions of the spanwise holes $(\mathrm{A}=$ amplitude of the pattern and $\lambda=$ wavelength of the pattern $)$

In practice, the oil container was fixed on the traversing mechanism movable in two axes. The mechanism was used to adjust the position of the wire with respect to the leading edge in order to attain a clear visualization of the vortex structure. Crossing down the test section, a small weight was tied at the end of the wire to make it straight. A DC power supply was connected to the ends of the wire to heat up the wire and generate smoke. The smoke in the boundary layer was illuminated by a green laser light sheet of $532 \mathrm{~nm}$ wavelength. The test models were placed in the middle of the wind tunnel test section. To record the flow field, a camera was positioned at a distance from the tunnel outlet. Figure 2 shows a schematic sketch of the wind tunnel indicating the position and orientation of the test model, camera and smoke visualization apparatus. The flow velocity must be kept above $1 \mathrm{~m} / \mathrm{s}$ to avoid buoyancy effect which resulted from the density difference of air and smoke. Since the Von Karman vortex-street occurs downstream of a circular cylinder at a Reynolds number beyond 45 the flow uniformity is affected that is not suitable for smoke-wire flow visualization (Cimbala et al. 1988; Mathieu and Scott 2000). Regarding the smoke-wire diameter used in the present study, the flow velocity is required to be lower than $4 \mathrm{~m} / \mathrm{s}$ corresponding to $\mathrm{Re}_{\mathrm{D}} \approx 50$. Therefore, $3 \mathrm{~m} / \mathrm{s}$ was chosen for the experiment.

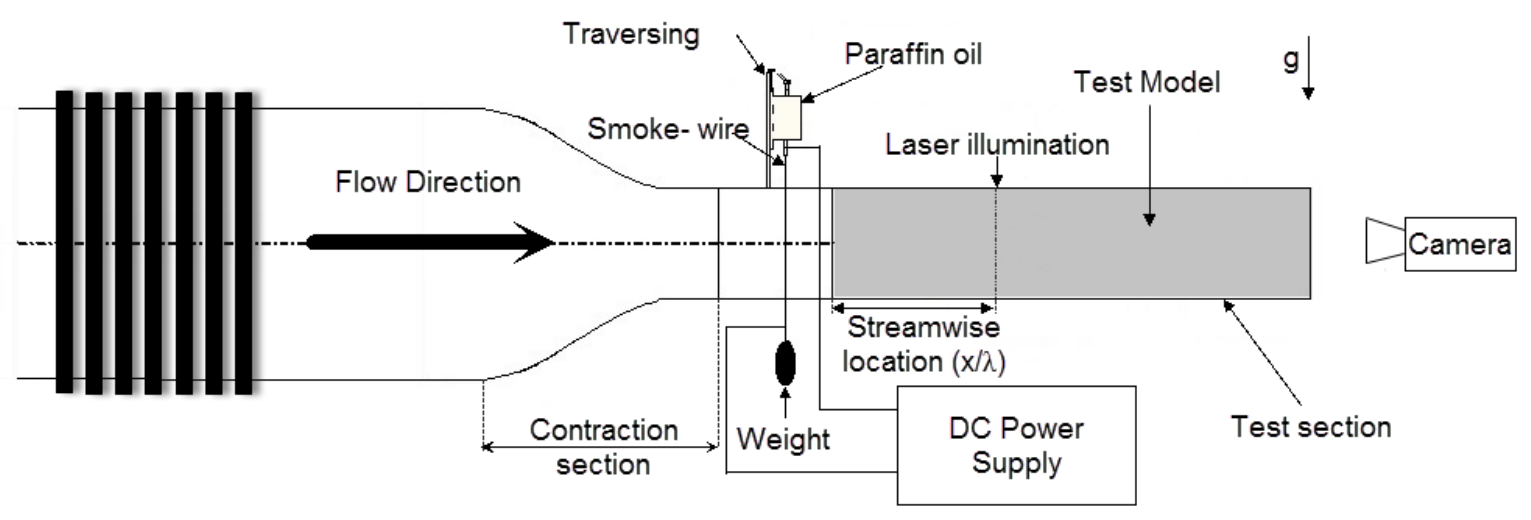

Fig. 2 Schematic of experimental setup

\section{Results and Discussion}

Visualization results were obtained at some stream wise locations from the flat plate leading edge for Reynolds number $\operatorname{Re}_{\lambda}$ based on wavelength $\lambda\left(\operatorname{Re}_{\lambda}=U_{\infty} \lambda / v\right)$ of 3080 corresponding to the freestream velocity $U_{\infty}=3 \mathrm{~m} / \mathrm{s}$. To investigate the vortex structures, the laser light sheet was shone normal to the surface at various distances at downstream of the leading edge. The vortex structure is examined in the presence of one spanwise array of holes, with spacing equal to wavelength $(\lambda)$, at location of $x / \lambda=2$ downstream of the troughs and peaks, separately. The range of Reynolds number and the streamwise distance of the visualized sections from the leading edge for tests can be seen in Table 1. Although the Reynolds number range and normalized streamwise distance provided in Table 1 are based on different criteria, only Reynolds number based on wavelength $\left(\operatorname{Re}_{\lambda}\right)$ and streamwise distance normalized by wavelength $(\mathrm{x} / \lambda)$ are used in the following sections due to the comparability of the scale of wavelength and counter-rotating streamwise vortices in the present work. 
Table 1 Test parameters and conditions

\begin{tabular}{|c|c|c|c|c|}
\hline \multicolumn{2}{|c|}{ Streamwise distance of the visualized sections } & \multicolumn{2}{c|}{ Reynolds number } \\
\hline $\mathrm{x}$ & $\mathrm{x} / \lambda$ & $\mathrm{x} / \mathrm{L}$ & $\mathrm{Re}_{\lambda}$ & $\mathrm{Re}_{\mathrm{L}}$ \\
\hline $10 \mathrm{~mm}$ & $2 / 3$ & $1 / 66$ & 3080 & 2053 \\
\hline $20 \mathrm{~mm}$ & $4 / 3$ & $2 / 66$ & 3080 & 4106 \\
\hline $30 \mathrm{~mm}$ & 2 & $3 / 66$ & 3080 & 6159 \\
\hline $100 \mathrm{~mm}$ & $20 / 3$ & $20 / 66$ & 3080 & 20533 \\
\hline $250 \mathrm{~mm}$ & $50 / 3$ & $25 / 66$ & 3080 & 51333 \\
\hline
\end{tabular}

where $\mathrm{L}=$ Plate length and $\lambda=$ wavelength.

\subsection{Formation and development of streamwise counter-rotating vortices}

Naturally, no streamwise counter-rotating vortices are formed over a flat surface with a plain leading edge. Installing perturbation wires just before the leading edge may lead to the appearance of these vortices over concave surfaces amplified by centrifugal instability (Peerhossaini and Bahri 1998; Mitsudharmadi et al. 2004). However, the leading edge pattern method is a more practical way to generate streamwise counter-rotating vortices (Budiman et al. 2014; Hasheminejad et al. 2014). In the present work, a triangular pattern (refer to Sec.2) is used at the flat plate leading edge to examine its effect on the development of such vortices. Fig. 3 shows the evolution of the spanwise distributed streamwise counter-rotating vortices at different streamwise locations of $x / \lambda=2 / 3,4 / 3$ and 2 . In fact, pairs of counter-rotating vortices appeared downstream of the trough pattern. At location $x / \lambda=2 / 3$ as shown in Fig. 3(a), the structure of the vortices is clearly visible. As the vortices evolve downstream, the vortex structures become unclear (Fig. 3b) and eventually they completely break down prior to turbulence (Fig. 3c).

As schematically shown in Fig. 4, the flow downstream of the troughs of the triangular pattern, containing low momentum fluid, moves upward, away from the surface until it encounters the higher momentum fluid in the freestream. The low momentum fluid is then deflected downward to the surface resulting in the engulfment of the high momentum fluid at the downwash region. The entrainment of the high momentum fluid into the boundary layer due to the formation of the streamwise vortices enhances mixing. The entire motion of the fluid is discernable in Fig. 3(a) as a dome covering two roundish lobes split by a stem. This type of structure is then called as "mushroom-like" structure. The appearance of the stem and hat of the mushroom-like structure are indicative of the fluid moving away from (upwash) and returning back to (downwash) the wall, respectively.

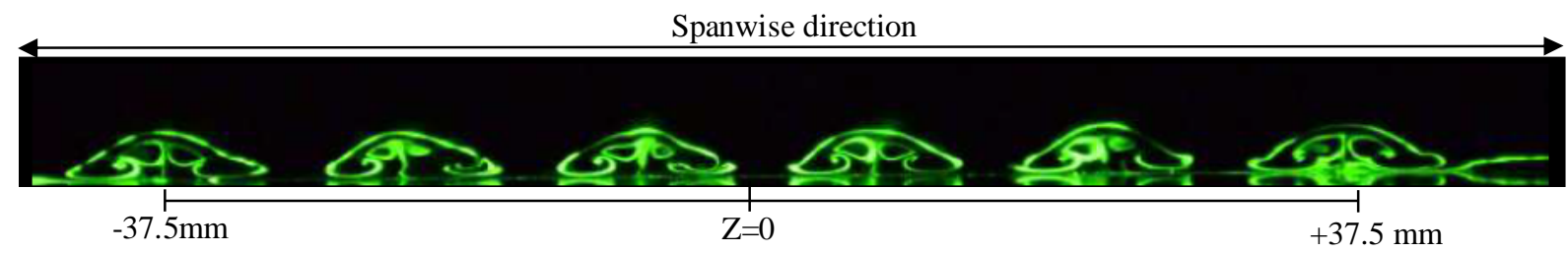

(a) at $\mathrm{x}=2 / 3 \lambda(=10 \mathrm{~mm})$

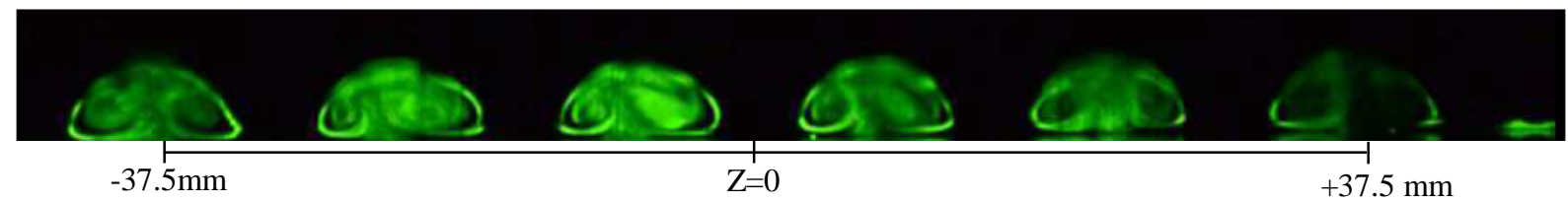

(b) at $x=4 / 3 \lambda(=20 \mathrm{~mm})$

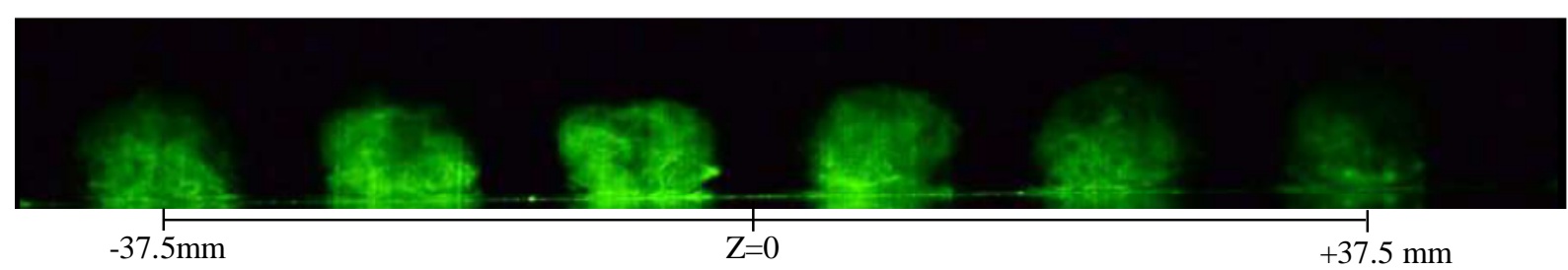

(c) at $\mathrm{x}=2 \lambda(=30 \mathrm{~mm})$

Fig. 3 Cross-sectional views of streamwise counter-rotating vortices for the reference plate at $\operatorname{Re}_{\lambda}=3080$ for some streamwise positions 


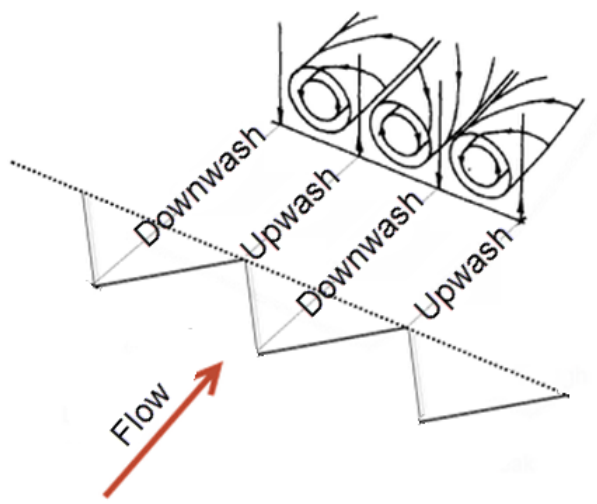

Fig. 4 Schematic of the formation of streamwise counter-rotating vortices on a flat plate with triangular leading edge pattern

\subsection{Effect of the holes on the vortex structure and flow stability}

When the fluid flow passes the reference plate, the introduced disturbances are exhibited in the form of mushroom-like structures. The vortices with the same wavelength as the leading edge pattern are diffused and break down into turbulence earlier than the Blasius flow. If the identical experimental conditions are applied on Plate A the same result is observed. As presented in Fig. 5 the visualization results for Plate A, obtained at the same locations as those shown in Fig. 3, reveal that the streamwise counter-rotating vortices are generated immediately after the trough and manifest themselves as mushroom-like structures. The clear mushroom-like structures shown in Fig. 5a may indicate that the boundary layer is still laminar. As the growth of the disturbances become more prominent, the structure fades away further downstream and eventually breaks down into turbulence. The visualizations results presented in Figs. 3 and 5 show that the vortices are qualitatively alike at the same cross sections in many aspects such as its configuration, size, inner structure and the breakdown to turbulence location. Therefore, it can be inferred that placing holes at the upwash region (after the trough) does not significantly alter the vortices structure and their evolution.

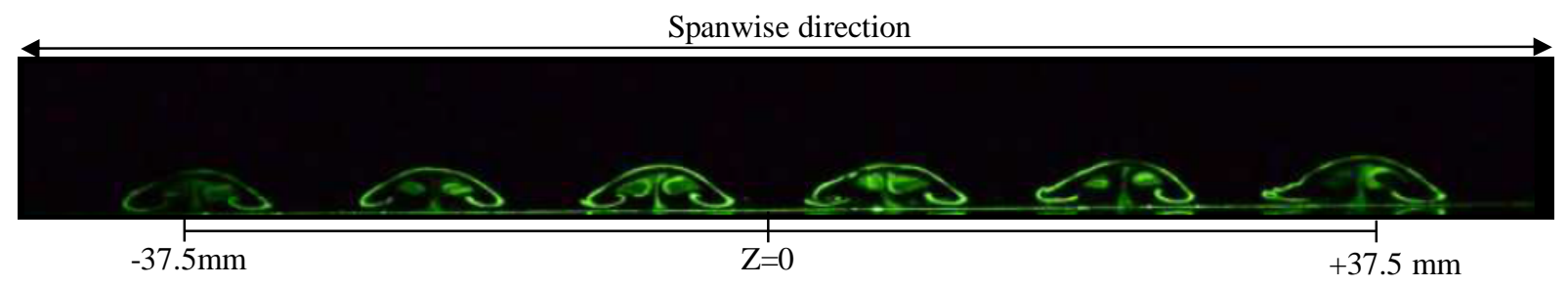

(a) at $\mathrm{x}=2 / 3 \lambda(=10 \mathrm{~mm})$

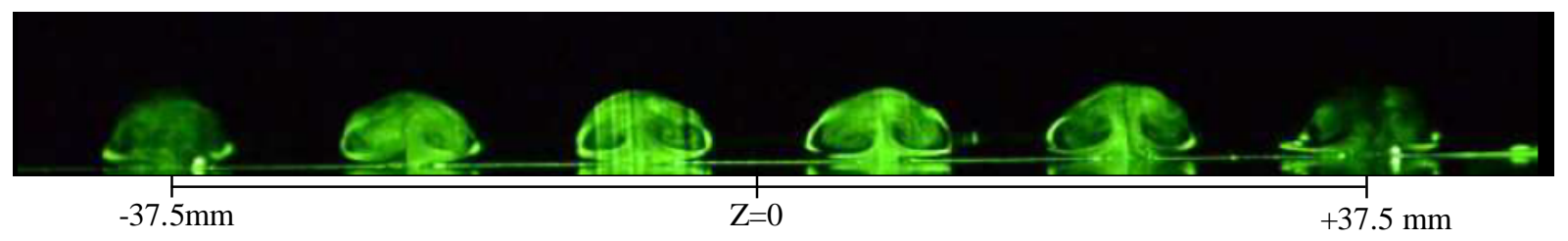

(b) at $\mathrm{x}=4 / 3 \lambda(=20 \mathrm{~mm})$

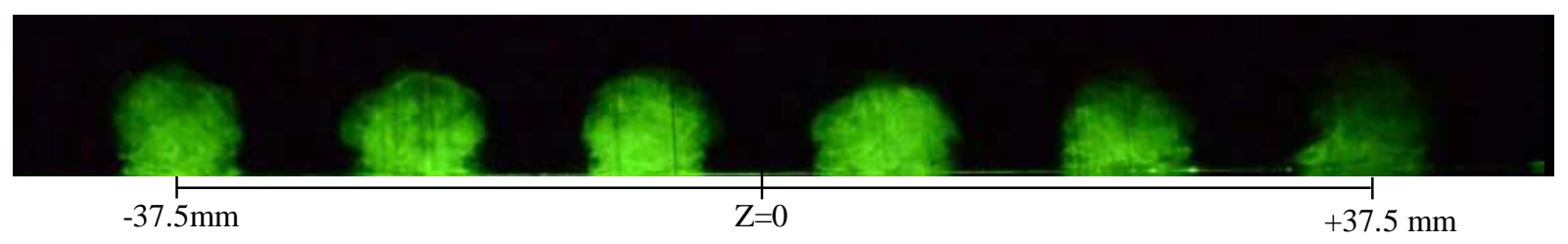

(c) at $\mathrm{x}=2 \lambda(=30 \mathrm{~mm})$

Fig. 5 Cross-sectional views of streamwise counter-rotating vortices for Plate A at $\operatorname{Re}_{\lambda}=3080$ for some streamwise positions 
On the contrary, the findings for Plate B show that the holes downstream of the peaks can strikingly affect the vortex structure and its stability. Fig. 6 displays the appearance and evolution of the vortices along the plate at locations of $\mathrm{x} / \lambda=4 / 3,2,20 / 3$ and 50/3. Similar to the earlier plates, the mushroom-like vortices are generated after the trough but in a smaller size and grow upward while is traveling in streamwise direction. Unlike the visualization results presented in Figs. 3 and 5, at $x / \lambda=4 / 3$ the vortex structures remain clear (Fig. 6(a)). As the vortices evolve further, the presence of the holes downstream of the peaks of the triangular pattern on plate $B$, causes the vortex structures to sustain until $x / \lambda=50 / 3$. As presented in Figs. 6(c) and 6(d), the formation of mushroom lobes is evident prior to $x / \lambda=50 / 3$ where the vortices reach their maximum height and then extend further downstream while the growth of the mushroom-like vortices is almost halted. It is also shown that the vortices structures are tilted to the left at $x / \lambda=50 / 3$ (Fig. $6(\mathrm{~d})$ ). The visualization results presented in Fig. 6 could imply that the presence of the holes downstream of peaks, at downwash region, has a suction effect which draws the low momentum fluid towards the wall. As shown in Fig. 7, the smoke streaks past over the pattern peaks are sucked into the holes. The suction at holes implies that the pressure at the top surface of the plate is higher than that at the other side. The pressure difference might be attributed to the tapered leading edge. In other words, holes decrease the boundary layer thickness and consequently stabilize the boundary layer (Becker and Jovanovic 2010). Furthermore, the fact that the vortex structures remain clear until the last section of visualization may also indicate that the presence of the holes downstream of the triangular pattern extends the laminar streamwise vortices and transition to turbulence which may be beneficial in some applications. The current study also shows that the breakdown of the vortices can be delayed by more than eight times longer than those of the reference plate and plate A. Since visualization results cannot give more information to understand better the phenomenon of the tilted vortices, a quantitative study will be carried out in the future.

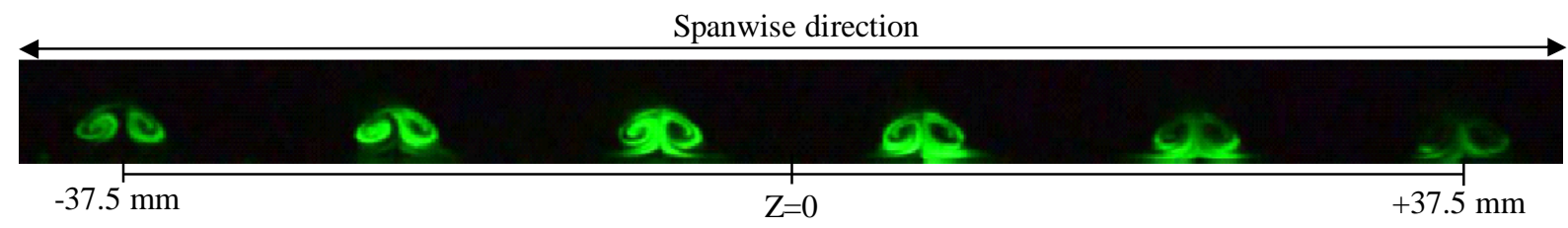

(a) at $\mathrm{x}=4 / 3 \lambda(=20 \mathrm{~mm})$

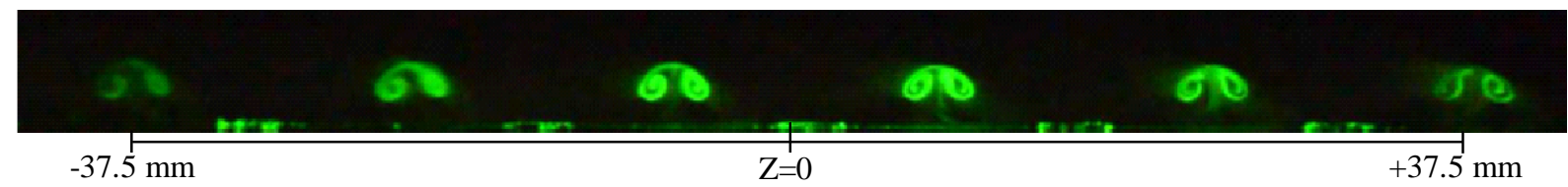

(b) at $\mathrm{x}=2 \lambda(=30 \mathrm{~mm})$

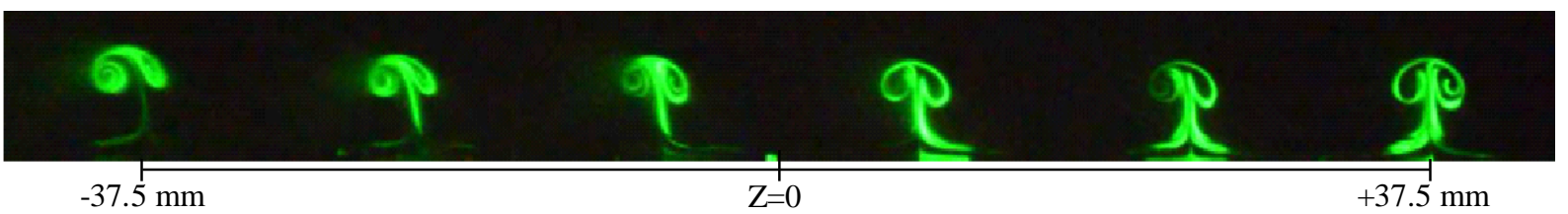

(c) at $\mathrm{x}=20 / 3 \lambda(=100 \mathrm{~mm})$

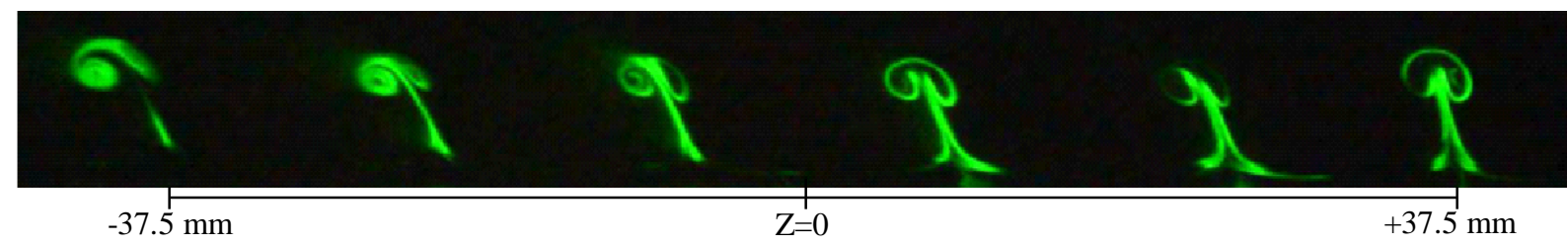

(d) at $\mathrm{x}=50 / 3 \lambda(=250 \mathrm{~mm})$

Fig. 6 Cross-sectional views of streamwise counter-rotating vortices for Plate $B$ at $\operatorname{Re}_{\lambda}=3080$ for some streamwise positions 


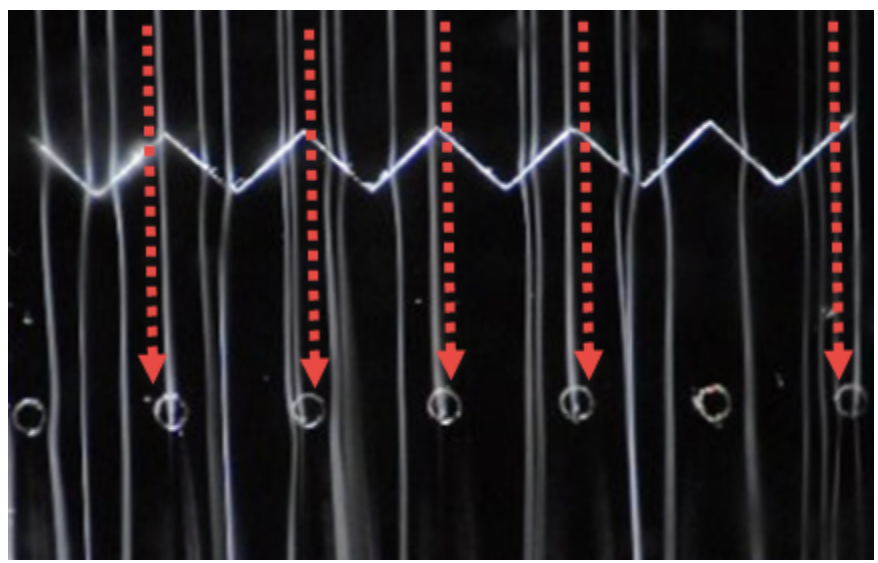

Fig. 7 Suction of the smoke streaks in to the holes (Top surface)

To show how leading edge pattern affect the boundary layer, Table 2 is provided to compare the approximate vortex height (h) with the Blasius boundary layer thickness $(\delta)$. As tabulated, the vortex heights are larger than the Blasius boundary layer thicknesses, as in the case of vortices produced by vortex generators. This implies that the boundary layer fluid is lifted at upwash region by the vortices and then entrained downward into the boundary layer. There are also other analogies between the leading edge patterns and the counter-rotating vortex generators. For example, both of them produce streamwise vortices which increase in size further downstream (Godard and Stanislas 2006).

Additionally, in case of counter-rotating vortex generators, the low momentum region (or upwash region) occurs in the space between vortex generators while the high momentum region is in the symmetry plane of the vertex generator. This manner of flow distribution is comparable to what is performed by tubercles or pattern peaks on the leading edge (Hansen et al. 2011; Wei et al. 2015), therefore, it can be concluded that each peak of the pattern can be used as a passive flow control similar to a counter-rotating vortex generator.

Table 2 Comparisons of vortex height with Blasius boundary layer thickness at various streamwise locations

\begin{tabular}{|c|c|c|c|c|}
\hline \multirow{2}{*}{ Streamwise location (x) } & \multirow{2}{*}{ Blasius boundary layer thickness $(\delta)$} & \multicolumn{3}{|c|}{ Vortex height (h) } \\
\cline { 3 - 5 } & & Reference plate & Plate A & Plate B \\
\hline $10 \mathrm{~mm}$ & $1.10 \mathrm{~mm}$ & $3.4 \mathrm{~mm}$ & $2.9 \mathrm{~mm}$ & - \\
\hline $20 \mathrm{~mm}$ & $1.56 \mathrm{~mm}$ & $5.3 \mathrm{~mm}$ & $4.6 \mathrm{~mm}$ & $2.9 \mathrm{~mm}$ \\
\hline $30 \mathrm{~mm}$ & $1.91 \mathrm{~mm}$ & $6.8 \mathrm{~mm}$ & $5.7 \mathrm{~mm}$ & $3.6 \mathrm{~mm}$ \\
\hline $100 \mathrm{~mm}$ & $3.49 \mathrm{~mm}$ & - & - & $5.5 \mathrm{~mm}$ \\
\hline $250 \mathrm{~mm}$ & $5.52 \mathrm{~mm}$ & - & - & $6.5 \mathrm{~mm}$ \\
\hline
\end{tabular}

\section{Conclusions and recommendations}

Streamwise counter-rotating vortices generated by a leading edge variation were visualized using smoke-wire flow visualization technique. The leading edge variation with a triangular pattern generated streamwise counter-rotating vortices in the form of mushroom-like structures which are initiated from the troughs. The mushroom-like structures consist of a pair of counter-rotating vortices streamwise propagated over the flat plate under zero-pressure condition. For the reference plate, the vortex structures start being diffused at $x / \lambda=4 / 3$ and then break down to turbulence at $x / \lambda=2$ downstream of the leading edge. The flow visualization results indicated that placing holes downstream of the troughs (plate A) does not affect the evolution of the vortices. However, the presence of the holes downstream of the peaks stabilized the vortex structures within the boundary layer. It delays the breakdown of the vortex structures by more than 8 times longer than those of reference plate and plate A. The suction effect due to the holes draws low momentum fluid toward the wall and allows the streamwise counter-rotating vortices to be propagated downstream in the form of mushroom-like structures which are clear but tilted aside. The mechanism of the vortex evolution for this case was not known yet. Detailed quantitative measurements will be carried out to further study the effect of the holes in the evolution of such vortices.

Acknowledgments The authors would like to thank all the laboratory technologists in the Fluid Mechanics Laboratory, Department of Mechanical Engineering, National University of Singapore for their help in conducting the experiments and also Dr. Yann Bouremel of Institute of Ophthalmology, University College, London, UK for his help and advice in flow visualization. 


\section{References}

Aider J-L, Duriez T, Wesfreid JE (2008) From natural to forced counter-rotating streamwise vortices in boundary layers. J Phys Conf Ser 137:012009. doi: 10.1088/1742-6596/137/1/012009

Becker S, Jovanovic J (2010) Investigations of Suction in a Transitional Flat-Plate Boundary Layer. In: Schlatter P, Henningson DS (eds) Seventh IUTAM Symposium on Laminar-Turbulent Transition SE - 76. Springer Netherlands, pp 465-468

Budiman AC, Mitsudharmadi H, Bouremel Y, Winoto SH, Low HT (2014) Visualization of pre-set vortices in boundary layer flow over wavy surface in rectangular channel. J Vis Vol. 18(4):pp. 669-677. doi: $10.1007 / \mathrm{s} 12650-014-0252-\mathrm{z}$

Cimbala JM, Nagib HM, Roshko A (1988) Large structure in the far wakes of two-dimensional bluff bodies. J Fluid Mech 190:265. doi: 10.1017/S0022112088001314

Cranston B, Laux C, Altman A (2012) Leading Edge Serrations on Flat Plates at Low Reynolds Number. In: 50th AIAA Aerospace Sciences Meeting including the New Horizons Forum and Aerospace Exposition. American Institute of Aeronautics and Astronautics,

Fish FE, Battle JM (1995) Hydrodynamic design of the humpback whale flipper. J Morphol 225:51-60. doi: 10.1002/jmor. 1052250105

Fish FE, Weber PW, Murray MM, Howle LE (2011) The tubercles on humpback whales' flippers: application of bio-inspired technology. Integr Comp Biol 51:203-213. doi: 10.1093/icb/icr016

Godard G, Stanislas M (2006) Control of a decelerating boundary layer. Part 1: Optimization of passive vortex generators. Aerosp Sci Technol 10:181-191. doi: 10.1016/j.ast.2005.11.007

Hansen KL, Kelso RM, Dally BB (2011) Performance Variations of Leading-Edge Tubercles for Distinct Airfoil Profiles. AIAA J 49:185-194. doi: 10.2514/1.j050631

Hasheminejad SM, Mitsudharmadi H, Winoto SH (2014) Effect of Flat Plate Leading Edge Pattern on Structure of Streamwise Vortices Generated in Its Boundary Layer. J Flow Control Meas Vis 02:18-23. doi: 10.4236/jfcmv.2014.21004

Johari H, Henoch CW, Custodio D, Levshin A (2007) Effects of Leading-Edge Protuberances on Airfoil Performance. AIAA J 45:2634-2642. doi: 10.2514/1.28497

Lin JC, Howard FG, Selby G V. (1991) Exploratory study of vortex-generating devices for turbulent flow separation control. In: 29th Aerospace Sciences Meeting. American Institute of Aeronautics and Astronautics,

Mathieu J, Scott J (2000) An introduction to turbulent flow.

Miklosovic DS, Murray MM, Howle LE (2007) Experimental Evaluation of Sinusoidal Leading Edges. J Aircr 44:1404-1408. doi: 10.2514/1.30303

Miklosovic DS, Murray MM, Howle LE, Fish FE (2004) Leading-edge tubercles delay stall on humpback whale (Megaptera novaeangliae) flippers. Phys Fluids 16:L39. doi: 10.1063/1.1688341

Mitsudharmadi H, Winoto SH, Shah DA (2004) Development of boundary-layer flow in the presence of forced wavelength Görtler vortices. Phys Fluids 16:3983. doi: 10.1063/1.1789771

Nadesan T, Mitsudharmadi H, Lee TS, Winoto SH (2014) Quasi-streamwise counter-rotating vortices generated by convergent riblets in flat plate boundary layer. J Vis Vol. 17:pp. 319-325. doi: 10.1007/s12650-014-0210-9

Peerhossaini H, Bahri F (1998) On the spectral distribution of the modes in nonlinear Gortler instability. Exp Therm Fluid Sci 16:195-208. doi: 10.1016/S0894-1777(97)10029-2

Soderman PT (1972) Aerodynamic effects of leading-edge serrations on a two-dimensional arifoil. United States

Stein B, Murray MM (2005) Stall Mechanism Analysis of Humpback Whale Flipper Models. In: Proceedings of Unmanned Untethered Submersible Technology (UUST), UUST05. NH,

Tandiono, Winoto SH, Shah DA (2009) Visualizing shear stress in gortler vortex flow. J Vis 12:195-202. doi: 10.1007/BF03181858

Van Nierop E a., Alben S, Brenner MP (2008) How bumps on whale flippers delay stall: An aerodynamic model. Phys Rev Lett 100:54502. doi: 10.1103/PhysRevLett.100.054502

Velte CM, Hansen MOL, Jønck K (2007) Experimental and numerical investigation of the performance of vortex generators on separation control. J Phys Conf Ser 75:012030. doi: 10.1088/1742-6596/75/1/012030

Webb RL, Kim N-H (2005) Principles of Enhanced Heat Transfer, 2nd edn. Taylor \& Francis, Great britain

Wei Z, New TH, Cui YD (2015) An experimental study on flow separation control of hydrofoils with leading-edge tubercles at low Reynolds number. Ocean Eng 108:336-349. doi: 10.1016/j.oceaneng.2015.08.004

Winoto SH, Low HT (1991) Transition of boundary layer flows in the presence of Goertler vortices. Exp Fluids 10:281-284. doi: 10.1007/BF00202461

Winoto SH, Mitsudharmadi H, Shah DA (2005) Visualizing Görtler vortices. J Vis 8:315-322. doi: 10.1007/BF03181550 